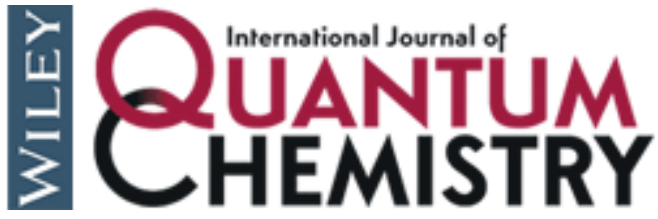

\title{
A comparative multi-reference configuration interaction study of the low-lying states of two thione isomers of thiophenol.
}

\begin{tabular}{|r|l|}
\hline Journal: & International Journal of Quantum Chemistry \\
\hline Manuscript ID & QUA-2020-0067.R1 \\
\hline Wiley - Manuscript type: & Full Paper \\
\hline Author: & n/a \\
\hline Complete List of Authors: & $\begin{array}{l}\text { de Lima, Fillipe; Universidade Federal da Paraiba, Departamento de } \\
\text { Química } \\
\text { Pereira Rodrigues, Gessenildo; Universidade Federal da Paraíba, } \\
\text { Departamento de Química } \\
\text { Lucena Jr., Juracy; Universidade Estadual da Paraíba, química } \\
\text { Do Monte, Elizete Ventura; Universidade Federal da Paraíba, } \\
\text { Departamento de Química } \\
\text { Fausto, Rui; Universidade de Coimbra, química } \\
\text { Reva, Igor; University of Coimbra, Department of Chemistry } \\
\text { do Monte, Silmar; Universidade Federal da Paraíba, Departamento de } \\
\text { Química; Universidade Federal da Paraiba, Química }\end{array}$ \\
\hline Keywords: & $\begin{array}{l}\text { multi-reference configuration interaction, thione isomers of thiophenol, } \\
\text { excited states }\end{array}$ \\
\hline & \\
\hline
\end{tabular}

\section{SCHOLARONE \\ Manuscripts}




\title{
A comparative multi-reference configuration interaction study of the low-lying states of two thione isomers of thiophenol
}

Filipe Belarmino de Lima, ${ }^{1}$ Gessenildo Pereira Rodrigues, ${ }^{2}$ Juracy Regis de Lucena Junior, ${ }^{3}$ Elizete Ventura, ${ }^{1}$ Rui Fausto, ${ }^{4}$ Igor Reva, ${ }^{4 *}$ Silmar Andrade do Monte ${ }^{1 *}$

${ }^{1}$ Departamento de Química, CCEN, Universidade Federal da Paraíba, 58059-900, João Pessoa-PB, Brazil

${ }^{2}$ Faculdade Rebouças, 58406-040, Campina Grande-PB, Brazil

${ }^{3}$ Departamento de Química, Universidade Estadual da Paraíba, 58429-500, Campina GrandePB, Brazil

${ }^{4}$ CQC-Departamento de Química, Universidade de Coimbra, 3004-535, Coimbra, Portugal *Corresponding authors: reva@qui.uc.pt, silmar@quimica.ufpb.br

\begin{abstract}
Multi-reference configuration interaction, MR-CI (including extensivity corrections, named $+Q$ ) calculations have been performed on $\mathrm{S}_{0}$ to $\mathrm{S}_{3}$ states of cyclohexa-2,4diene-1-thione (thione 24) and cyclohexa-2,5-diene-1-thione (thione 25), which are thione isomers of thiophenol. Several types of uncontracted MR-CIS and MR-CISD wavefunctions have been employed, comprising MR-CI expansions as large as $\sim 365 \times 10^{6}$ configuration state functions. The nature of the studied excited states has been characterized. Vertical excitation energies $(\Delta \mathrm{E})$ and oscillator strengths $(f)$ have been computed. The most intense transitions $\left(\mathrm{S}_{0} \rightarrow \mathrm{S}_{2}\right.$ for $\mathbf{2 4}$ and $\mathrm{S}_{0} \rightarrow \mathrm{S}_{3}$ for $\mathbf{2 5}$ ) do not change with the wavefunction, although a variation as large as $\sim 1 \mathrm{eV}$ has been obtained for the $\mathrm{S}_{3}$ state of $\mathbf{2 4}$, at the highest (MR-CI+Q) level. On the other hand, $\Delta \mathrm{E}$ changes at most $\sim 0.56 \mathrm{eV}$ for $\mathbf{2 5}$, as the wavefunction changes, at the same level. The $S_{1}$ state of both thiones has $n \pi^{*}$ character and is in the visible region. For $24 S_{2}$ and $S_{3}$ are $\pi \pi^{*}$ and $n \pi *$ states, respectively, while for $\mathbf{2 5}$ the reverse order has been obtained. $\mathrm{S}_{2}$ and $\mathrm{S}_{3}$ are in the range from $\sim 3.5$ to $5.2 \mathrm{eV}$, again at the highest level. It is the first time that the excited states of the title molecules are studied. The computed results agree with the experimental onsets of photoreactions of thiones $\mathbf{2 4}$ and $\mathbf{2 5}$ found by Reva et. al. (Phys. Chem. Chem. Phys. 2015, 17, 4888).
\end{abstract}




\section{Introduction}

Cyclohexa-2,4-diene-1-thione (24; Figure 1) has been identified in a ground state isomerization reaction of thiophenol, studied at the MP2 and QCISD(T) levels by AlMuhtaseb et al. ${ }^{[1]}$ Those authors obtained a very large barrier of $\sim 63 \mathrm{kcal} / \mathrm{mol}$ for the thiophenol $\rightarrow \mathbf{2 4}$ thiol-thione H-transfer reaction. Although formation of the analogous cyclohexa-2,5-diene-1-thione (25; see Figure 1) has also been studied, the authors did not find a direct pathway for formation of $\mathbf{2 5}$ from thiophenol. ${ }^{[1]}$

Reva et. al. ${ }^{[2]}$ have isolated thiophenol in cryogenic argon matrices and observed the reversible photochemical thiophenol $\leftrightarrow \mathbf{2 4}$ and $\mathbf{2 4} \leftrightarrow \mathbf{2 5}$ reactions. This was the first experimental observation of thione isomers $\mathbf{2 4}$ and 25. According to the authors, the direct thiophenol $\leftrightarrow \mathbf{2 5}$ photoisomerization reaction could not be discarded nor confirmed. ${ }^{[2]}$

In the present work the first three excited singlet states of $\mathbf{2 4}$ and $\mathbf{2 5}$ have been studied at the MR-CIS and MR-CISD levels, with inclusion of extensivity corrections (hereafter named $+Q$ ). Basis set effects were also taken into account. The results obtained for the two investigated systems were then used to address the excited states reached in the photochemical experiments performed by Reva et. al. ${ }^{[2]}$ This is the first time that the excited states of thiones $\mathbf{2 4}$ and $\mathbf{2 5}$ are studied.

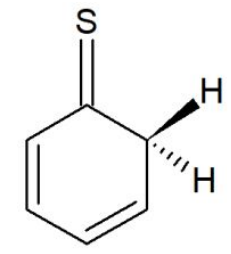

24

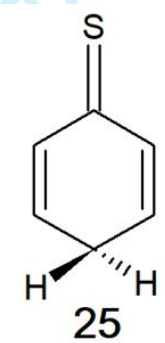

25

Figure 1. Thione isomers of thiophenol: cyclohexa-2,4-diene-1-thione (24, reads: "two-four") and cyclohexa-2,5-diene-1-thione (25, reads "two-five").

\section{Computational Methods}

The optimized structures of molecules $\mathbf{2 4}$ and $\mathbf{2 5}$ have been taken from ref. ${ }^{[2]}$ (B3LYP/aug-cc-pVTZ data). Frequency calculations have also been performed in ref. ${ }^{[2]}$, confirming that the obtained structures correspond to minima. Molecules 24 and 25 have $\mathrm{C}_{\mathrm{s}}$ and $\mathrm{C}_{2 \mathrm{v}}$ symmetry, respectively. 
In the present study, the active space used for thiones $\mathbf{2 4}$ and $\mathbf{2 5}$ at the CASSCF level (state average calculations) consists of 12 electrons in 11 orbitals. Two $\mathrm{A}^{\prime}$ and two $\mathrm{A}^{\prime \prime}$ states have been averaged at the CASSCF level for 24, while for $\mathbf{2 5}$ one state for each one of the four symmetries $\left(A_{1}, B_{1}, B_{2}\right.$ and $\left.A_{2}\right)$ has been averaged (in both cases with the same weights). For both systems these are the four lowest states at the CASSCF level. Due to the equivalence between the two $\mathrm{C}_{\mathrm{sp} 3}-\mathrm{H}$ bonds of $\mathbf{2 4}$ and $\mathbf{2 5}$, the $\sigma$ orbitals in these molecules are equally localized on these bonds. Two pairs of active $\sigma$ orbitals, the $\sigma_{\mathrm{C}-\mathrm{H}} / \sigma^{*}{ }_{\mathrm{C}-\mathrm{H}}$ and $\sigma_{\mathrm{CS}} / \sigma^{*}$ CS pairs, have been included for both $\mathbf{2 4}$ and $\mathbf{2 5}$. The $\mathrm{C}$ atom of the former pair is the $\mathrm{sp}^{3} \mathrm{C}$ atom, for both molecules (see Figure 1).

For 25, three of the six active $\pi$ orbitals are named $\pi_{C S}+\pi_{\text {ring }}\left(\equiv \pi_{1}\right), n_{\pi}\left(\equiv \pi_{3}\right.$, an essentially non-bonding $\mathrm{S}$ orbital) and $\pi_{C=C}$ (that is, mainly localized on the $\mathrm{C}=\mathrm{C}$ bonds, named $\pi_{2}$ ). The other non-bonding $S$ orbital, perpendicular to the previous one, is simply named $n$, and the three anti-bonding $\pi$ orbitals are named $\pi *_{C S}+\pi *_{\text {ring }}\left(\equiv \pi_{1}{ }^{*}\right), \pi_{2}{ }^{*}{ }_{C=C}\left(\equiv \pi_{2}{ }^{*}\right)$ and $\pi_{\text {ring }}^{*}\left(\equiv \pi_{3}^{*}\right)$.

For 24, a non-bonding $\mathrm{S}$ orbital is again named $n$ (as for $\mathbf{2 5}$ ). The three bonding active $\pi$ orbitals are named $\pi_{1}, \pi_{C S}\left(\equiv \pi_{2}\right)$ and $\pi_{S}+\pi_{C=C}\left(\equiv \pi_{3}\right)$, while the anti-bonding orbitals are named $\pi_{1}{ }^{*}{ }_{\text {ring }}+\pi^{*}{ }_{C S}\left(\equiv \pi_{1}{ }^{*}\right), \pi_{2}{ }^{*}{ }_{\text {ring }}+\pi^{*}{ }_{C S}\left(\equiv \pi_{2}{ }^{*}\right)$ and $\pi^{*}{ }_{C=C}\left(\equiv \pi_{3}{ }^{*}\right)$.

The subscripts of the orbitals (such as ${ }_{C S},{ }_{C}=C$, etc.) refer to their localization. Additional details can be found in the supporting information.

The $\sigma_{\mathrm{CH}} / \sigma^{*}$ CH pairs of $\mathbf{2 4}$ and $\mathbf{2 5}$ have been included in the active space (for most of the wavefunctions here used) as this study is the first step towards a description (on the same grounds) of photoinduced hydrogen migration pathways associated with the thiophenol $\rightarrow \mathbf{2 4}$, thiophenol $\rightarrow \mathbf{2 5}$ and $\mathbf{2 4} \rightarrow \mathbf{2 5}$ reactions. Besides, the above mentioned active space was constructed considering also the following two reasons: (i) as some preliminary calculations at the CASSCF level performed for thiophenol showed that the $\sigma_{\mathrm{CS}} / \sigma^{*} \mathrm{CS}$ pair should be included in the active space, due to its admixture with the $\sigma_{\mathrm{SH}} / \sigma^{*}{ }_{\mathrm{SH}}$ pair, the former pair has also been included in the active spaces of $\mathbf{2 4}$ and $\mathbf{2 5}$ (for most of the wavefunctions here used), due to the thiophenol $\rightarrow \mathbf{2 4}$ and thiophenol $\rightarrow \mathbf{2 5}$ reactions, ${ }^{[2]}$ which we intend to study; (ii) the highest level results for thiophenol (at the CASPT2 level, discussed later and compared to the results here obtained) include the eleven orbitals which transform into the eleven orbitals here used for $\mathbf{2 4}$ and $\mathbf{2 5}$, along the thiophenol $\rightarrow \mathbf{2 4}$ and thiophenol $\rightarrow \mathbf{2 5}$ reaction pathways (as will be discussed elsewhere). 
Five types of wavefunctions have been used in this work, named $w 1, w 11^{\prime}, w 2, w 3$ and $w 4$. $w 1$ and $w l^{\prime}$ are MR-CIS wavefunctions, while the remaining are MR-CISD wavefunctions. $w 1, w 1$ 'and $w 4$ are used for both systems. $w 2$ is only used for $\mathbf{2 5}$. For $w 2$ the CAS space has been reduced, transferring strongly and weakly occupied orbitals to the doubly occupied (DOCC) and auxiliary (AUX) spaces, respectively, as discussed below. $w 1^{\prime}$ is derived from $w 2$ simply reducing the internal $\rightarrow$ external excitation level from singles and doubles to singles only (see Table 1). For $w 3$ the CAS orbitals are split into reduced active space (RAS) and auxiliary (AUX) orbitals. It was not possible to use $w 3$ for $\mathbf{2 5}$, as in this case single occupied $\rightarrow$ anti-bonding excitations are not enough to generate the correct number of guess vectors for each irreducible representation. In the case of $w 4$ the four $\sigma$ orbitals have been removed from the CAS (at both CASSCF and MR-CI levels) and the $n$ and $\pi_{1}$ orbitals $\left(24 a^{\prime} / 9 b_{2}\right.$ and $3 a^{\prime \prime} / 3 b_{1}$, respectively, see the supporting information) have been transferred to the RAS space, and only single RAS $\rightarrow$ CAS excitations are allowed while generating the reference configuration state functions (CSFs), yielding a set of reference CSFs based on a CAS $(4,5)+$ single RAS $\rightarrow$ CAS excitations. Additional details concerning how these four wavefunctions are formed are given in Table 1.

$w 2$ is the largest MR-CISD wavefunction here used (see Table 1) and has only been employed for 25, due to its higher $\left(\mathrm{C}_{2 \mathrm{v}}\right)$ symmetry (see Figure 1), but at the expense of a reduced number of active orbitals. This reduction consists of transferring former active orbitals, namely, two bonding $\left(\sigma_{\mathrm{CS}}\right.$ and $\left.\sigma_{\mathrm{CH}}\right)$ and three anti-bonding orbitals $\left(\sigma_{\mathrm{CS}} *, \sigma_{\mathrm{CH}} *\right.$ and $\pi^{*}$ ring, see supporting information) to the DOCC and AUX spaces, respectively, at the MRCISD level.

The criteria chosen for these two sets of $w 2$ were: the active orbitals whose occupation numbers (nocc) are (at the CASSCF level) larger than 1.97 have been transferred to the DOCC space, while those with nocc $<0.1$ have been transferred to the AUX space, and only single CAS $\rightarrow$ AUX excitations are allowed (yielding a set of reference CSFs based on a $\mathrm{CAS}(8,6)+$ single CAS $\rightarrow$ AUX excitations, see Table 1). One can check the importance of double internal $\rightarrow$ external excitations through a comparison between the results obtained from $w l^{\prime}$ and $w 2$. On the other hand, a comparison between $w 1$ and $w l^{\prime}$ allows checking the effect of the reference wave function on the relative accuracy of the MR-CIS wavefunctions. 
Table 1. Wavefunctions used in this work, at the MR-CI level.

\begin{tabular}{c|l|l}
\hline Wavefunction & $\begin{array}{l}\text { excitation levels used to generate the reference } \\
\mathrm{CSFs}^{\mathrm{a}}\end{array}$ & $\begin{array}{l}\text { Internal } \rightarrow \text { external excitation } \\
\text { level/final MR-CI wavefunction }\end{array}$ \\
\hline \hline$w 1$ & $\mathrm{CAS}(12,11)$ & singles/MR-CIS \\
\hline$w 1^{\prime}$ & $\mathrm{CAS}(8,6)+$ single CAS $\rightarrow$ AUX excitations & singles/MR-CIS \\
\hline$w 2$ & $\mathrm{CAS}(8,6)+$ single CAS $\rightarrow$ AUX excitations & singles and doubles/MR-CISD \\
\hline$w 3^{c}$ & single occ $\rightarrow$ anti-bonding excitations & singles and doubles/MR-CISD \\
\hline$w 4$ & CAS $(4,5)+$ single RAS $\rightarrow$ CAS excitations & singles and doubles/MR-CISD \\
\hline
\end{tabular}

${ }^{a}$ Configuration State Functions; ' Internal corresponds to the set of doubly occupied + active + AUX orbitals, while external corresponds to the set of orbitals which are unoccupied (virtual) in the reference CSFs; ${ }^{\circ}$ For $w 3$ occ comprises the subset of six orbitals (active at the CASSCF level) with the highest occupation numbers, while the anti-bonding subset comprises the five active orbitals with the smallest occupation numbers. For additional details concerning these orbitals see text and supporting information.

Due to the size of the system and to the basis sets used, multi-reference configuration interaction calculations with single and double excitations (MR-CISD) are only feasible for 24 with a large reduction of the number of reference CSFs, at least for uncontracted MR-CI wavefunctions (as discussed later). Such reduction has been applied to yield the wavefunctions $w 3$ and $w 4$ (see Table 1). The first one has been devised with the purpose to see what is the net effect of reducing the internal excitation level (in other words, splitting the CAS into RAS + AUX orbitals) but at the same time increasing the internal $\rightarrow$ external excitation level, yielding an MR-CISD wavefunction. $w 3$ has a similar size to that of $w 1$. Again, due to the size of the system, an internal excitation level larger than one (between the occ and the anti-bonding subsets described in Table 1), combined with single and double internal $\rightarrow$ external excitations (yielding a MR-CISD wavefunction), is computationally prohibitive for $\mathbf{2 4}$. However, the relatively small number of active orbitals used in $w 4$ allows all excitations compatible with a $\mathrm{CAS}(4,5)$. Besides, as explained before, additional reference CSFs are also generated through RAS $\rightarrow$ CAS excitations (see Table 1). w4 corresponds to the largest CI expansion used for 24.

Once the reference CSFs were formed, they were used to generate the excited CSF through single excitations from all internal into all external orbitals, at the MR-CIS level (for $w 1$ and $w l^{\prime}$, see Table 1), and through single and double internal $\rightarrow$ external excitations at the MR-CISD level (for $w 2, w 3$ and $w 4$, see Table 1). The final CSF space is formed by the reference along with the excited CSFs. 
There are two subsets of low-lying orbitals at the ground state geometries, the frozen core (FC) and the doubly occupied valence orbitals, at the MR-CIS/MR-CISD levels. The FC subset comprises the $\mathrm{K}$ (for the $\mathrm{C}$ atom) and $\mathrm{K}+\mathrm{L}$ (for the $\mathrm{S}$ atom) shells. Such choice for the FC is based on the essentially correct description of the excited states of other systems containing another third row atom, $\mathrm{Cl}$, at the MR-CISD level. ${ }^{[3-10]}$ The difference between doubly occupied and FC orbitals is that, while the former set remains doubly occupied only in the reference CSFs, the latter is kept as so in the reference as well as in the excited CSF space. The multireference extension of the Davidson correction $(+Q)$ has been used to take the sizeextensivity error into account. ${ }^{[11-13]}$ Rigorously speaking, the Davidson correction should only be used for CISD/MR-CISD wavefunctions, not for CIS/MR-CIS wavefunctions. ${ }^{[13]}$ According to eqs. (42) and (43) from ref. [13] it is clear that the stabilization due to the Davidson correction is always larger for an MR-CISD wavefunction, as compared to that for an MR-CIS wavefunction formed from the same set of reference CSFs. This is due to (i) the sum of the weights of the reference CSFs is always smaller in the MR-CISD than in the MRCIS wavefunction; (ii) the final MR-CISD energy is always smaller than the final MR-CIS energy. Thus, as the final MR-CISD energy is always smaller than the final MR-CIS energy, the Davidson corrected MR-CISD energy is always smaller than the Davidson corrected MRCIS energy. However, error cancellation effects can lead to similar Davidson corrections for the excitation energies computed with MR-CIS and MR-CISD wavefunctions (which is the case for the $w 2$ and $w l^{\prime}$ calculations performed for $\mathbf{2 5}$, as discussed later). As such error cancellation effects are not guaranteed, one should be cautious while using the Davidson correction formula for MR-CIS wavefunctions.

The interactive space restriction ${ }^{[14]}$ has been used for $w 2$ and $w 4$ (see Table 1). All CASSCF, MR-CISD, and MR-CISD+Q calculations have been performed with the COLUMBUS program system. ${ }^{[15-18]}$ The atomic orbitals (AO) integrals used by COLUMBUS have been computed with the DALTON program. ${ }^{[19]}$ The aug-cc-pVTZ and the mixed aug-cc$\mathrm{pVDZ}(\mathrm{C}, \mathrm{H}) / \mathrm{aug}-\mathrm{cc}-\mathrm{pVTZ}(\mathrm{S})$ basis sets have been used in this study. ${ }^{[20-22]}$

\section{Results and discussion}

\section{Basis set effect}

The basis set effect has been taken into account at the CASSCF level, for both systems (see Table 2), and at the $w 1$ and $w 4$ levels for 25 (see Table 3). As it can be seen from Tables 2 and 3 , the $\Delta \mathrm{E}$ values obtained with both basis sets differ by at most $0.03 \mathrm{eV}$. Besides, the 
main configurations obtained for all four states do not change with the basis set, and the differences between their weights are virtually negligible, at CASSCF, $w 1$ and $w 4$ levels. Besides, the differences between the $f$ values obtained with the two basis sets are small, at the $w 1$ and $w 4$ levels (see Table 3). Therefore, the results shown in Tables 2 and 3 give us confidence about the reliability of the aug-cc-pVDZ $(\mathrm{C}, \mathrm{H}) /$ aug-cc-pVTZ(S) basis set towards the computationally much more demanding aug-cc-pVTZ(C,H,S) basis set.

Table 2. $\triangle \mathrm{E}$ values (in $\mathrm{eV}$ ) and configuration weights computed at the CASSCF level with the aug-cc-pVDZ(C,H)/aug-cc-pVTZ(S) basis set, for $\mathbf{2 4}$ and 25 thione isomers.

\begin{tabular}{|c|c|c|c|c|c|}
\hline \multicolumn{3}{|r|}{24} & \multicolumn{3}{|r|}{25} \\
\hline State & $\Delta \mathrm{E}$ & Configurations ${ }^{\mathrm{a}, \mathrm{b}}$ & State & $\Delta \mathrm{E}$ & Configurations $\mathrm{s}^{\mathrm{a}, \mathrm{b}}$ \\
\hline $1^{1} \mathrm{~A}^{\prime}$ & 0.00 & $0.60 \mathrm{gs}+0.25 \pi_{3} \pi_{1} *$ & $1^{1} \mathrm{~A}_{1}$ & 0.00 & $0.75 \mathrm{gs}+0.10 \mathrm{n}_{\pi} \pi_{1} *$ \\
\hline $1^{1} \mathrm{~A}^{\prime \prime}$ & $\begin{array}{l}2.09 \\
(2.07)^{\mathrm{c}}\end{array}$ & $0.80 \mathrm{n} \pi_{1}^{*}$ & $1^{1} \mathrm{~A}_{2}$ & $\begin{array}{l}2.18 \\
(2.16)^{\mathrm{c}}\end{array}$ & $0.83 \mathrm{n} \pi_{1}^{*}$ \\
\hline $2^{1} \mathrm{~A}^{\prime}$ & $\begin{array}{c}4.50 \\
(4.49)^{\mathrm{c}} \\
\end{array}$ & $\begin{array}{l}0.21 \pi_{2} \pi_{1}^{*}+0.19 \pi_{3} \pi_{1}^{*}+ \\
0.15 \pi_{3}^{0} \pi_{1}^{* 2}+0.12 \pi_{3} \pi_{2}^{*}\end{array}$ & $1^{1} \mathrm{~B}_{1}$ & $\begin{array}{c}4.98 \\
(4.95)^{\mathrm{c}}\end{array}$ & $0.45 n \pi_{2}^{*}+0.29 n^{1} \pi_{2}^{1} \pi_{1}^{* 2}$ \\
\hline $2^{1} \mathrm{~A}^{\prime \prime}$ & $\begin{array}{c}4.55 \\
(4.53)^{\mathrm{c}} \\
\end{array}$ & $\begin{array}{c}0.44 \mathrm{n} \pi_{2}{ }^{*}+0.23 \mathrm{n}^{1} \pi_{3}{ }^{1} \pi_{1}{ }^{* 2}+ \\
0.12 \mathrm{n}^{1} \pi_{2}{ }^{1} \pi_{1}{ }^{* 2}\end{array}$ & $1^{1} \mathrm{~B}_{2}$ & $\begin{array}{c}5.36 \\
(5.35)^{\mathrm{c}} \\
\end{array}$ & $\begin{array}{c}0.28 \mathrm{n}_{\pi} \pi_{2}{ }^{*}+0.27 \pi_{2}{ }^{1} \mathrm{n}_{\pi}{ }^{1} \pi_{1}{ }^{* 2} \\
+0.19 \pi_{2} \pi_{1}{ }^{*}\end{array}$ \\
\hline
\end{tabular}

${ }^{\mathrm{a} g s}$ stands for the ground state configuration; only configurations with weights larger than 0.1 are shown; ${ }^{b}$ Configurations $a^{0} b^{2}$ and $a^{1} b^{1} c^{2}$ correspond to the $a \rightarrow b$ and $(a, b) \rightarrow c$ double excitations, respectively, and the remaining configurations correspond to single excitations; ${ }^{c}$ Results obtained with the aug-cc-pVTZ $(\mathrm{C}, \mathrm{H}, \mathrm{S})$ basis set. The weights obtained with both basis sets differ by at most 0.01 .

\section{Vertically excited states of 24 and 25}

$\Delta \mathrm{E}$ values, configuration weights and oscillator strengths $(f)$ computed with $w 1, w 2$, $w l^{\prime}$ and $w 4$ for 25 are shown in Table 3. As it can be seen through comparison between Tables 2 and 3, inclusion of dynamic electron correlation (at both MR-CIS and MR-CISD levels) changes the $\Delta \mathrm{E}$ values by at most $0.41 \mathrm{eV}$. A decrease in the $\Delta \mathrm{E}$ values is obtained upon inclusion of extensivity correction, but the effect is smaller than that of the dynamic electron correlation, leading to a maximum change of $0.19 \mathrm{eV}$. The nature of all studied states is the same, at the CASSCF and MR-CI levels (compare Tables 2 and 3). It is important to point out the non-negligible contribution of configurations formed by double excitations to the $\pi_{1}{ }^{*}$ orbital, in the $1^{1} \mathrm{~B}_{1}$ and $1^{1} \mathrm{~B}_{2}$ states, although these weights decrease upon inclusion of dynamic electron correlation (compare Tables 2 and 3 ). 
An important difference between the results obtained for thione isomers $\mathbf{2 4}$ and $\mathbf{2 5}$ and those obtained for the thiol isomer (thiophenol) is the absence of configurations formed by excitations to the $\sigma^{*}$ orbital, a feature observed for the $S_{2}$ state of thiophenol. ${ }^{[23-27]}$ However, such difference is expected, as in thiophenol this orbital is localized on a considerably weaker (S-H) bond.

Using the CASPT2/aug-cc-pVTZ results for thiophenol as reference ${ }^{[23]}(4.3,4.5$ and 5.1 $\mathrm{eV}$ for the $\mathrm{S}_{1}, \mathrm{~S}_{2}$ and $\mathrm{S}_{3}$ states, respectively), one can see a decrease in the excitation energies (as one goes from thiophenol to 25), with the largest effect obtained for the first excited state, followed by the second excited state. Compared to the highest level $w 2(+\mathrm{Q})$ results (as discussed later), the decrease for $\mathrm{S}_{1}$ is as large as $\sim 2.1 \mathrm{eV}$, while for $\mathrm{S}_{2}$-one has an increase of only $\sim 0.3 \mathrm{eV}$, at the same level. For $\mathrm{S}_{3}$ the changes are almost negligible, at both $w 2(+\mathrm{Q})$ and $w 4(+\mathrm{Q})$ levels. For $w 1$ and $w l^{\prime}$ decreases of $\sim 0.3 \mathrm{eV}$ are obtained (see Table 3 ). It is important to stress that one should be cautious while comparing results obtained with different methods, for different molecules, as part of the difference is likely to be due to the methods. Nevertheless, as a much larger effect has been observed for $\mathrm{S}_{1}$, it is very likely that at least in this case the obtained trend is correct.

It is interesting to point out the change of nature of $S_{1}$ of the thione isomer $\mathbf{2 5}$ as compared to the thiol form of thiophenol. Although there is some contribution of the $n$ orbital (perpendicular to the $\pi$ system of the ring) to the $\sigma_{\mathrm{SH}}$ and $\sigma_{\mathrm{SC}}$ orbitals of thiophenol, ${ }^{[24]}$ configurations containing excitations from these orbitals are absent in the lowest four excited states of thiophenol. ${ }^{[23-27]}$ On the other hand, in $\mathbf{2 5}$ (and also in 24, as discussed later) the $n$ orbital is very well localized (as shown in the supporting information). While in thiophenol $\mathrm{S}_{1}$ is a $\pi \pi^{*}$ state, in 25 it is an $n \pi^{*}$ state (see Table 3). $S_{2}$ is also an $n \pi^{*}$ state, and $S_{3}$ is a $\pi \pi^{*}$ state (see Table 3). Thus, only the nature of $S_{3}$ is the same both in thiophenol ${ }^{[23]}$ and in the thione isomer 25.

The first excited state of $\mathbf{2 5}\left(1^{1} \mathrm{~A}_{2}\right)$ is in the visible region (with $\Delta \mathrm{E}=2.15$ or $2.36 \mathrm{eV}$, at the $w 2(+\mathrm{Q})$ and $w 4(+\mathrm{Q})$ levels, respectively), but the $1^{1} \mathrm{~A}_{1} \rightarrow 1^{1} \mathrm{~A}_{2}$ transition is dipole forbidden, by symmetry. However, the experimental threshold required for photochemical transformations of $\mathbf{2 5}, \lambda<332 \mathrm{~nm}(3.73 \mathrm{eV})$, is consistent with a transition within the first band (see Table 3). Therefore, some intensity gain is expected to be taking place around 3.73 $\mathrm{eV}$, due to vibronic and/or spin-orbit coupling mechanisms. Besides, one cannot rule out some intensity borrowing from the nearby $\mathrm{S}_{0} \rightarrow \mathrm{S}_{2}$ transition. 
Table 3. $\Delta \mathrm{E}$ values (in $\mathrm{eV}$ ), configuration weights and oscillator strengths $(f)$ computed for 25, at the $w 1, w 2, w l^{\prime}$ and $w 4$ levels (see Table 1), with the aug-cc-pVDZ(C,H)/aug-cc$\mathrm{pVTZ}(\mathrm{S})$ basis set. Multireference extension of the size-extensivity Davidson correction is indicated by $(+Q)$.

\begin{tabular}{|c|c|c|c|c|}
\hline & \multicolumn{4}{|c|}{ Wavefunction $w 1$} \\
\hline States & $\Delta \mathrm{E}$ & $\Delta \mathrm{E}(+\mathrm{Q})$ & $f\left(\times 10^{3}\right)$ & configurations $^{\mathrm{a}, \mathrm{b}}$ \\
\hline $1{ }^{1} \mathrm{~A}_{1}$ & 0.00 & 0.00 & -- & $0.74 \mathrm{gs}$ \\
\hline $1^{1} \mathrm{~A}_{2}$ & $2.04(2.03)^{\mathrm{c}}$ & $2.00(1.99)^{\mathrm{c}}$ & $--^{-d}$ & $0.80 \mathrm{n} \pi_{1}^{*}$ \\
\hline $1^{1} \mathrm{~B}_{1}$ & $4.65(4.62)^{\mathrm{c}}$ & $4.50(4.47)^{c}$ & $0.557(0.524)^{\mathrm{c}}$ & $0.60 \mathrm{n} \pi_{2}{ }^{*}+0.14 \mathrm{n}^{1} \pi_{2}^{1} \pi_{1} *^{2}$ \\
\hline \multirow[t]{2}{*}{$1^{1} \mathrm{~B}_{2}$} & $4.98(4.97)^{\mathrm{c}}$ & $4.84(4.82)^{\mathrm{c}}$ & $20.062(19.688)^{\mathrm{c}}$ & $\begin{array}{c}0.35 \pi_{2} \pi_{1}^{*}+0.22 \mathrm{n}_{\pi} \pi_{2}^{*}+ \\
0.21 \mathrm{n}_{\pi}{ }^{1} \pi_{2}{ }^{1} \pi_{1} *^{2}\end{array}$ \\
\hline & \multicolumn{4}{|c|}{ Wavefunction $w 2$} \\
\hline States & $\Delta \mathrm{E}$ & $\Delta \mathrm{E}(+\mathrm{Q})$ & $f\left(\times 10^{3}\right)$ & configurations $^{\mathrm{a}, \mathrm{b}}$ \\
\hline $1{ }^{1} \mathrm{~A}_{1}$ & 0.00 & 0.00 & -- & $0.65 \mathrm{gs}$ \\
\hline $1^{1} \mathrm{~A}_{2}$ & 2.12 & 2.15 & $-\mathrm{-O}^{\mathrm{d}}$ & $0.72 \mathrm{n} \pi_{1}^{*}$ \\
\hline $1^{1} \mathrm{~B}_{1}$ & 4.90 & 4.82 & 0.098 & $0.46 \mathrm{n} \pi_{2}{ }^{*}+0.20 \mathrm{n}^{1} \pi_{2}{ }^{1} \pi_{1} *^{2}$ \\
\hline \multirow[t]{2}{*}{$1^{1} \mathrm{~B}_{2}$} & 5.17 & 5.07 & 15.326 & $\begin{array}{c}0.25 \mathrm{n}_{\pi} \pi_{2}{ }^{*}+0.22 \pi_{2} \pi_{1} *+ \\
0.21 \mathrm{n}_{\pi}{ }^{1} \pi_{2}{ }^{1} \pi_{1} *^{2} \\
\end{array}$ \\
\hline & \multicolumn{4}{|c|}{ Wavefunction $w l^{\prime}$} \\
\hline States & $\Delta \mathrm{E}$ & $\Delta \mathrm{E}(+\mathrm{Q})$ & $f\left(\times 10^{3}\right)$ & configurations $\mathrm{s}^{\mathrm{a}, \mathrm{b}}$ \\
\hline $1{ }^{1} \mathrm{~A}_{1}$ & 0.00 & 0.00 & -- & $0.74 \mathrm{gs}$ \\
\hline $1^{1} \mathrm{~A}_{2}$ & 2.08 & 2.08 & $--^{d}$ & $0.80 \mathrm{n} \pi_{1}^{*}$ \\
\hline $1^{1} \mathrm{~B}_{1}$ & 4.76 & 4.63 & 0.649 & $0.60 \mathrm{n} \pi_{2}{ }^{*}+0.14 \mathrm{n}^{1} \pi_{2}{ }^{1} \pi_{1} *^{2}$ \\
\hline \multirow[t]{2}{*}{$1^{1} \mathrm{~B}_{2}$} & 4.95 & 4.79 & 25.929 & $\begin{array}{c}0.34 \pi_{2} \pi_{1}^{*}+0.22 \mathrm{n}_{\pi} \pi_{2}^{*+} \\
0.21 \mathrm{n}_{\pi}{ }^{1} \pi_{2}{ }^{1} \pi_{1}{ }^{2}\end{array}$ \\
\hline & \multicolumn{4}{|c|}{ Wavefunction $w 4$} \\
\hline States & $\Delta \mathrm{E}$ & $\Delta \mathrm{E}(+\mathrm{Q})$ & $f\left(\times 10^{3}\right)$ & configurations ${ }^{\mathrm{a}, \mathrm{b}}$ \\
\hline $1^{1} \mathrm{~A}_{1}$ & 0.00 & 0.00 & -- & $0.65 \mathrm{gs}$ \\
\hline $1^{1} \mathrm{~A}_{2}$ & $2.47(2.45)^{\mathrm{c}}$ & $2.36(2.36)^{\mathrm{c}}$ & $-{ }^{\mathrm{d}}$ & $0.74 \mathrm{n} \pi_{1}^{*}$ \\
\hline $1^{1} \mathrm{~B}_{1}$ & $5.25(5.23)^{\mathrm{c}}$ & $5.06(5.05)^{\mathrm{c}}$ & $0.115(0.092)^{\mathrm{c}}$ & $0.51 \mathrm{n} \pi_{2}{ }^{*}+0.19 \mathrm{n}^{1} \pi_{2}{ }^{1} \pi_{1} *^{2}$ \\
\hline $1^{1} \mathrm{~B}_{2}$ & $5.18(5.17)^{\mathrm{c}}$ & $5.07(5.06)^{\mathrm{c}}$ & $16.395(15.180)^{\mathrm{c}}$ & $\begin{array}{c}0.26 \mathrm{n}_{\pi} \pi_{2}{ }^{*}+0.22 \mathrm{n}_{\pi}{ }^{1} \pi_{2}{ }^{1} \pi_{1} *^{2}+ \\
0.21 \pi_{2} \pi_{1}{ }^{*}\end{array}$ \\
\hline
\end{tabular}

ags stands for the ground state configuration; only configurations with weights larger than 0.1 are shown. The weights obtained with both basis sets (for $w 1$ ) differ by at most 0.01 ; ${ }^{b}$ Configurations $a^{1} b^{1} c^{2}$ correspond to the $(a, b) \rightarrow c$ double excitations, while the remaining configurations correspond to single excitations; 'Results obtained with the aug-ccpVTZ(C,H,S) basis set; 'Forbidden by symmetry. For $w l$ and $w l^{\prime}$ the total MR-CIS/MR$\mathrm{CIS}+\mathrm{Q}$ energies are (in au) -628.524468/-628.542458 and -628.470817/-628.487837, respectively; For $w 2$ and $w 4$ the total MR-CISD/MR-CISD+Q energies are -629.081146/629.2309174 and -629.079709/-629.229106, respectively. 
Due to the judicious choice of the CASSCF active orbitals to be transferred to the DOCC and AUX spaces while forming the reference set of CSFs for $w 2$ (see Table 1 and the previous discussion concerning the criteria used for this transfer), it is expected that $w 2$ already recovers a large fraction of the electron correlation of an MR-CISD wavefunction formed from a set of CAS $(12,11)$ reference CSFs. Consequently, the results obtained with $w 2$ can be considered the most reliable ones for $\mathbf{2 5}$. As already mentioned, $w 2$ is the largest MRCI wavefunction here employed, achieving $3.6 \times 10^{8} \mathrm{CSFs}$.

From what has been said one can test the reliability of $w l^{\prime}$ for $\mathbf{2 5}$ comparing its results with those obtained from $w 2$. Such comparison allows addressing the importance of the double internal $\rightarrow$ external excitations (see Table 1). As it can be seen from Table 3, the computed excitation energies are close, with a maximum difference of $0.28 \mathrm{eV}$, obtained for $1^{1} \mathrm{~B}_{2}$ including extensivity corrections. The main configurations are maintained, for $w l^{\prime}$ and $w 2$, and the largest change obtained for the configuration weights is 0.14 (for $1{ }^{1} \mathrm{~B}_{1}$ ), a value which can be considered relatively small. On the other hand, the effect on the $f$ values, due to the change from $w l^{\prime}$ to $w 2$, is significant, with reductions down to $\sim 15$ and $59 \%$ for $1{ }^{1} \mathrm{~B}_{1}$ and $1^{1} B_{2}$, respectively (see Table 3). Despite such reduction, the $S_{0} \rightarrow S_{3}$ transition remains the most intense one for both wavefunctions. Therefore, $w l^{\prime}$ can be considered a relatively good approximation to $w 2$ (a computationally much more demanding wavefunction). Besides, due to the error cancellation effects mentioned previously, the size-extensivity correction in the $\Delta$ E values computed with $w l^{\prime}$ and $w 2$ are similar (see Table 3 ).

As can be seen from Table 3 the effect associated with the change from $w 1$ to $w l^{\prime}$ is smaller than that between $w 2$ and $w l^{\prime}$, for the $\Delta \mathrm{E}$ and $f$ values. $\Delta \mathrm{E}$ values change by at most $0.13 \mathrm{eV}$, and the $f$ values increase $\sim 16$ and $29 \%$ for the $\mathrm{S}_{0} \rightarrow \mathrm{S}_{2}$ and $\mathrm{S}_{0} \rightarrow \mathrm{S}_{3}$ transitions, respectively, while changing the wavefunction from $w 1$ to $w 1$ '. The main configurations remain the same as well as their weights virtually don't change upon the change from $w 1$ to $w l^{\prime}$ (see Table 3).

Through a comparison between $w 2$ and $w 4$ one can verify how good the latter as an approximation to the former is. Without extensivity correction the largest difference between the $\Delta \mathrm{E}$ values computed with $w 2$ and $w 4$ is $0.35 \mathrm{eV}$. Upon inclusion of extensivity correction one has slightly smaller differences, of at most $0.24 \mathrm{eV}$. Interestingly, either with or without extensivity correction the difference between the $\Delta \mathrm{E}$ values of $\mathrm{S}_{3}$, obtained from $w 2$ or $w 4$, is virtually negligible (see Table 3). As the wavefunction changes from $w 2$ to $w 4$ the $f$ values increase only $\sim 15$ and $7 \%$ for the $\mathrm{S}_{0} \rightarrow \mathrm{S}_{2}$ and $\mathrm{S}_{0} \rightarrow \mathrm{S}_{3}$ transitions, respectively. Again, the 
main configurations are maintained and their weights change by at most 0.05 , from $w 2$ to $w 4$ (see Table 3). Thus, $w 4$ can be considered a good approximation to $w 2$, especially if extensivity correction is included.

$\Delta \mathrm{E}$ values, configuration weights and oscillator strengths $(f)$ computed with $w 1, w 1{ }^{\prime}, w 3$ and $w 4$ for $\mathbf{2 4}$ are shown in Table 4. It is clear that the effect of dynamic electron correlation is considerably larger for the $\Delta \mathrm{E}$ values of $\mathbf{2 4}$ than for those of $\mathbf{2 5}$ (especially for $\mathrm{S}_{2}$ and $\mathrm{S}_{3}$ ) and the effect for the latter is slightly dependent on the state (compare Table 2 with Tables 3 and 4). On the other hand, for $\mathbf{2 4}$ the effect of dynamic electron correlation is highly dependent on the state and on the wavefunction. For instance, as for the $2^{1} \mathrm{~A}^{\prime}\left(\mathrm{S}_{2}\right)$ state computed with $w 1$ a reduction of $0.68 \mathrm{eV}$ is obtained, for the same state calculated with $w 3$ and $w 4$ one has reductions of only 0.24 and $0.19 \mathrm{eV}$, respectively, upon inclusion of dynamic electron correlation. However, in the case of the fourth state $\left(2^{1} \mathrm{~A}^{\prime \prime}\right)$ a much larger change is obtained for $w 3$, as compared to that obtained for $w 1$ (0.92 versus $0.23 \mathrm{eV})$. In contrast, for $w 4$ one has a slight increase of $0.28 \mathrm{eV}$ (compare the CASSCF and MR-CI results from Tables 2 and 4, respectively).

Albeit the nature of the excited states (that is, $n \pi^{*}$ or $\pi \pi^{*}$ ) of $\mathbf{2 4}$ does not change upon inclusion of dynamic electron correlation, the multiconfigurational character of $\mathrm{S}_{2}$ and $\mathrm{S}_{3}$ decreases significantly as dynamic electron correlation is included (compare Tables 2 and 4).

In the case of the $\mathrm{S}_{1}$ state of $\mathbf{2 4}$ and $\mathbf{2 5}$ the effect of extensivity correction is similar and small, changing the $\Delta \mathrm{E}$ values by at most 0.11 and $0.12 \mathrm{eV}$, for $\mathbf{2 5}$ and $\mathbf{2 4}$, respectively, both obtained with $w 4$. For the $\mathrm{S}_{2}$ and $\mathrm{S}_{3}$ states of $\mathbf{2 4}$ the effect is significantly larger, decreasing the $\Delta \mathrm{E}$ values of $\mathrm{S}_{2}$ and $\mathrm{S}_{3}$ by at most 0.32 and $0.22 \mathrm{eV}$, respectively (see Table 4).

It is important to point out that, even considering only single internal $\rightarrow$ external excitations from the reference CSFs generated at the CAS(12,11) level (as in $w 1$, see Table 1), for 24 the final number of CSFs is already very large, $\sim 2.1 \times 10^{8} \mathrm{CSFs}$, due to the system size, number of active orbitals and basis set. Therefore, if one includes single and double excitations, the calculation becomes computationally prohibitive, at least for an uncontracted multi-reference CI wavefunction, which is the type of MR-CI wavefunctions used in this work. ${ }^{[28]}$ The same can be said even if one uses the same type of active space reduction based on the CASSCF occupation numbers, as it has been done for the wavefunction $w 2$ of 25 . 
Table 4. $\Delta \mathrm{E}$ values (in $\mathrm{eV}$ ), configuration weights and oscillator strengths $(f)$ computed for 24, at the $w 1, w 1$, w3 and $w 4$ levels (see Table 1), with the aug-cc-pVDZ(C,H)/aug-cc$\mathrm{pVTZ}(\mathrm{S})$ basis set. Multireference extension of the size-extensivity Davidson correction is indicated by $(+Q)$.

\begin{tabular}{|c|c|c|c|c|}
\hline & \multicolumn{4}{|c|}{ Wavefunction $w 1$} \\
\hline States & $\Delta \mathrm{E}$ & $\Delta \mathrm{E}(+\mathrm{Q})$ & $f\left(\times 10^{3}\right)$ & configurations $^{\mathrm{a}, \mathrm{b}}$ \\
\hline $1^{1} \mathrm{~A}^{\prime}$ & 0.00 & 0.00 & -- & $0.62 \mathrm{gs}+0.19 \pi_{3} \pi_{1}{ }^{*}$ \\
\hline $1^{1} \mathrm{~A}^{\prime \prime}$ & 1.92 & 1.88 & 0.052 & $0.79 \mathrm{n} \pi_{1}^{*}$ \\
\hline $2^{1} \mathrm{~A}^{\prime}$ & 3.82 & 3.54 & 248.8 & $0.54 \pi_{3} \pi_{1}^{*}+0.16 \mathrm{gs}$ \\
\hline \multirow[t]{2}{*}{$2^{1} \mathrm{~A}^{\prime \prime}$} & 4.32 & 4.22 & 0.061 & $0.52 \mathrm{n} \pi_{2}{ }^{*}+0.18 \mathrm{n}^{1} \pi_{3}{ }^{1} \pi_{1}{ }^{* 2}$ \\
\hline & \multicolumn{4}{|c|}{ Wavefunction $w l^{\prime}$} \\
\hline States & $\Delta \mathrm{E}$ & $\Delta \mathrm{E}(+\mathrm{Q})$ & $f\left(\times 10^{3}\right)$ & configurations $\mathrm{s}^{\mathrm{a}, \mathrm{b}}$ \\
\hline $1^{1} \mathrm{~A}^{\prime}$ & 0.00 & 0.00 & -- & $0.63 g s+0.19 \pi_{3} \pi_{1} *$ \\
\hline $1^{1} \mathrm{~A}^{\prime \prime}$ & 1.95 & 1.96 & 0.052 & $0.81 \mathrm{n} \pi_{1}^{*}$ \\
\hline $2^{1} \mathrm{~A}^{\prime}$ & 3.88 & 3.56 & 293.0 & $0.57 \pi_{3} \pi_{1}^{*}+0.18 \mathrm{gs}$ \\
\hline \multirow[t]{2}{*}{$2^{1} \mathrm{~A}^{\prime \prime}$} & 4.38 & 4.31 & 0.073 & $0.5 \ln \pi_{2}{ }^{*}+0.20 \mathrm{n}^{1} \pi_{3}{ }^{1} \pi_{1}{ }^{* 2}$ \\
\hline & \multicolumn{4}{|c|}{ Wavefunction $w 3$} \\
\hline States & $\Delta \mathrm{E}$ & $\Delta \mathrm{E}(+\mathrm{Q})$ & $f\left(\times 10^{3}\right)$ & configurations $\mathrm{s}^{\mathrm{a}, \mathrm{b}}$ \\
\hline $1^{1} \mathrm{~A}^{\prime}$ & 0.00 & 0.00 & -- & $0.61 \mathrm{gs}+0.16 \pi_{3} \pi_{1}{ }^{*}$ \\
\hline $1^{1} \mathrm{~A}^{\prime \prime}$ & 2.27 & 2.36 & 0.053 & $0.77 \mathrm{n} \pi_{1}^{*}$ \\
\hline $2^{1} \mathrm{~A}^{\prime}$ & 4.26 & 4.06 & 317.1 & $0.53 \pi_{3} \pi_{1}^{*}+0.13 \mathrm{gs}$ \\
\hline \multirow[t]{2}{*}{$2^{1} \mathrm{~A}^{\prime \prime}$} & 5.47 & 5.25 & 0.033 & $0.76 \mathrm{n} \pi_{2}^{*}$ \\
\hline & \multicolumn{4}{|c|}{ Wavefunction $w 4$} \\
\hline States & $\Delta \mathrm{E}$ & $\Delta \mathrm{E}(+\mathrm{Q})$ & $f\left(\times 10^{3}\right)$ & configurations $\mathrm{s}^{\mathrm{a}, \mathrm{b}}$ \\
\hline $1^{1} \mathrm{~A}^{\prime}$ & 0.00 & 0.00 & -- & $0.56 \mathrm{gs}+0.16 \pi_{3} \pi_{1}{ }^{*}$ \\
\hline $1^{1} \mathrm{~A}^{\prime \prime}$ & 2.31 & 2.19 & 0.041 & $0.73 n \pi_{1}^{*}$ \\
\hline $2^{1} \mathrm{~A}^{\prime}$ & 4.31 & 4.09 & 179.8 & $0.32 \pi_{3} \pi_{1}^{*}+0.13 \pi_{2} \pi_{1}^{*}$ \\
\hline $2^{1} \mathrm{~A}^{\prime \prime}$ & 4.83 & 4.62 & 0.001 & $0.46 n \pi_{2}{ }^{*}+0.19 n^{1} \pi_{3}{ }^{1} \pi_{1}{ }^{* 2}$ \\
\hline
\end{tabular}

${ }^{\mathrm{a}} \mathrm{gs}$ stands for the ground state configuration; only configurations with weights larger than 0.1 are shown; ${ }^{b}$ Configurations $a^{1} b^{1} c^{2}$ correspond to the $(a, b) \rightarrow c$ double excitations, while the remaining configurations correspond to single excitations. For $w 1, w 1^{\prime}, w 3$ and $w 4$ the total MR-CI/MR-CI+Q energies are (in au) -628.521163/-628.539777, -628.466399/-628.484396, $629.050072 /-629.212969$ and $-629.073981 /-629.225881$, respectively. 
One very important point is what type of wavefunction recovers a larger fraction of the electron correlation provided by a complete MR-CISD wavefunction, that is, an MR-CISD wavefunction based on a set of CAS $(12,11)$ reference CSFs. As already mentioned, such type of calculation is not affordable, at least for an uncontracted wavefunction. However, one has two affordable options: (i) only single internal $\rightarrow$ external excitations, yielding an MR-CIS wavefunction ( $w 1$, see Table 1); (ii) an MR-CISD wavefunction based on reference CSFs generated through limited excitations among the internal orbitals (from the occ to the antibonding orbitals described in Table 1), as in the case of $w 3$ (for which only single occ $\rightarrow$ antibonding excitations have been applied, see Table 1). Though the answer concerning which type of wavefunction, $w 1$ or $w 3$, recovers a larger fraction of the electron correlation provided by a complete MR-CISD wavefunction could not be obtained, one can compare the effect due to the change from $w 1$ to $w 3$ on the properties here studied for 24 (see Table 4). As case (ii) calculations based on higher than single excitations are not affordable, one could not study the effect of the excitation level (within the internal orbitals given in Table 1) on the final properties. Instead, one can study the effect of removing the $\sigma$ orbitals and, at the same time, increasing the excitation level within the active orbitals, through comparison between $w 3$ and $w 4$ (see Table 1).

As can be seen from Table 4, there are relatively large differences between the $\Delta \mathrm{E}$ results obtained with $w 1$ and with the MR-CISD wavefunctions ( $w 3$ and $w 4$, see Table 1), varying from 0.31 to $1.15 \mathrm{eV}$, and the maximum difference has been obtained for $\mathrm{S}_{3}\left(2^{1} \mathrm{~A}^{\prime \prime}\right)$ either with or without extensivity corrections. On the other hand, the differences between the $\Delta \mathrm{E}$ values obtained with $w 3$ and $w 4$, for $\mathrm{S}_{1}\left(1^{1} \mathrm{~A}^{\prime \prime}\right)$ and $\mathrm{S}_{2}\left(2^{1} \mathrm{~A}^{\prime}\right)$, are almost negligible (varying from 0.03 to $0.17 \mathrm{eV}$, see Table 4). For $\mathrm{S}_{3}$ the differences between the $w 3$ and $w 4$ results are much larger (with values of 0.63 and $0.64 \mathrm{eV}$, with and without extensivity corrections, respectively, see Table 4). Therefore, it is clear that the $\Delta \mathrm{E}$ values obtained for $\mathrm{S}_{3}$ shows by far the largest average variation among the wavefunctions (see Table 4), which can be taken as an evidence of the greater difficulty in describing this state.

Similarly to what has been obtained for $\mathbf{2 5}$, the effect due to the change from $w 1$ to $w 1^{\prime}$ is small, leading to changes of at most 0.09 and $0.06 \mathrm{eV}$ in the $\Delta \mathrm{E}$ values, with and without extensivity corrections, respectively. The $f$ value of the $\mathrm{S}_{0} \rightarrow \mathrm{S}_{1}$ transition practically does not change, while those of the $\mathrm{S}_{0} \rightarrow \mathrm{S}_{2}$ and $\mathrm{S}_{0} \rightarrow \mathrm{S}_{3}$ transitions increase only 18 and $\sim 20 \%$, respectively (see Table 4). 
Based on the similarity between $\mathbf{2 4}$ and $\mathbf{2 5}$ and on the comparison between the results for 25, obtained from $w 2$ and $w 4$, it is expected that $w 4$ is a good approximation to $w 2$ also for 24, especially if extensivity correction is included, as aforementioned. Thus, $w 4$ is not only the largest wavefunction used for $\mathbf{2 4}$, but can also be considered the most reliable one. Consequently, the discrepancies between $w 1 / w l^{\prime}$ and $w 4$ are likely to be due to the unreliability of the former.

Despite the relatively large differences between the $\Delta \mathrm{E}$ values (especially for $\mathrm{S}_{3}$, see Table 4), the nature of the states does not change with the wavefunction, even though there are some significant variations of the multiconfigurational character of $S_{2}$ and $S_{3}$ (see Table 4). Nevertheless, although the multiconfigurational character of $\mathrm{S}_{2}$ is larger for $w 4$, the weight of the ground state configuration is virtually negligible in this case, differently of what happens for $w 1, w l^{\prime}$ and $w 3$ (see Table 4). For all three wavefunctions there are significant contributions of the $\pi_{3} \pi_{1}{ }^{*}$ configuration in the ground state wavefunction. For the $\mathrm{S}_{3}$ state one has significant contributions of a double excitation $\left(\mathrm{n}^{1} \pi_{3}{ }^{1} \pi_{1}{ }^{* 2}\right)$ only for $w 1$ and $w 4$ (see Table 4), which is expected, as the reference CSFs of $w 3$ do not include this double excitation (cf. Table 1).

For all three wavefunctions the most intense transition of $\mathbf{2 4}$ remains the same $\left(1^{1} \mathrm{~A}^{\prime} \rightarrow\right.$ $2^{1} \mathrm{~A}^{\prime}, \mathrm{S}_{0} \rightarrow \mathrm{S}_{2}$, see Table 4). Based on the results obtained for the $f$ values of 25 (and again based on the fact that $\mathbf{2 4}$ and $\mathbf{2 5}$ are similar), some decrease on the $f$ values of $\mathbf{2 4}$ is to be expected (due to the change from $w 4$ to $w 2$ ), if $w 2$ was not computationally prohibitive for 24 . However, it is not expected that the most intense transition changes. Such transition for $\mathbf{2 4}$ $\left(1^{1} \mathrm{~A}^{\prime} \rightarrow 2^{1} \mathrm{~A}^{\prime}\right)$ is approximately 11 times more intense than that of $25\left(1^{1} \mathrm{~A}_{1} \rightarrow 1^{1} \mathrm{~B}_{2}\right.$, compare the $w 4$ results in Tables 3 and 4). This information, along with their corresponding excitation energies of 4.09 and $5.07 \mathrm{eV}$ (obtained at the $w 4(+\mathrm{Q})$ level for $\mathbf{2 4}$ and 25, respectively, see Tables 3 and 4), can be helpful if one wants to discriminate these two isomers through UVVIS absorption spectroscopy.

By comparison between the CASPT2/aug-cc-pVTZ results for thiophenol, used as reference ${ }^{[23]}\left(4.3,4.5\right.$ and $5.1 \mathrm{eV}$ for the $\mathrm{S}_{1}, \mathrm{~S}_{2}$ and $\mathrm{S}_{3}$ states, respectively), and those obtained for $\mathbf{2 4}$, one can see how the $\Delta \mathrm{E}$ values change in the thiophenol vs. 24 pair. It is clear that the effect is by far the largest for $\mathrm{S}_{1}$ (as in the thiophenol vs. 25 pair). Comparing the CASPT2 results with the results obtained including extensivity corrections $(+Q)$, the excitation energies of $\mathrm{S}_{1}$ decrease by $\sim 2.1$ to $2.4 \mathrm{eV}$, depending on the wavefunction (see Table 4 ). With 
exception of $\mathrm{S}_{3}$ at the $w 3(+\mathrm{Q})$ level (where a slight increase of only $0.15 \mathrm{eV}$ is obtained), the other $\Delta \mathrm{E}(+\mathrm{Q})$ values of $\mathrm{S}_{2}$ and $\mathrm{S}_{3}$ decrease from 0.41 to $0.96 \mathrm{eV}$, depending on the state and on the level of theory used, again compared to the CASPT2 results (see Table 4).

The $\Delta \mathrm{E}(+\mathrm{Q})$ values of $\mathrm{S}_{1}$ (see Table 4 ), along with the computed $f$ values (although very small), are in line with the observation from ref. ${ }^{[2]}$ that $\mathbf{2 4}$ is the only isomer which absorbs in the visible region. As discussed before, even if the first excited state of $\mathbf{2 5}$ is also in the visible region, its transition dipole moment vanishes by symmetry.

The experimental threshold for the photochemical transformations of $\mathbf{2 4}(\lambda<427 \mathrm{~nm}=$ $2.90 \mathrm{eV}$ ) is consistent with excitation within the first band, associated with $1^{1} \mathrm{~A}^{\prime \prime}$ (an $\mathrm{n} \pi^{*}$ state; see Table 4). While in thiophenol $S_{1}$ is a $\pi \pi^{*}$ state, in 24 it is an $n \pi^{*}$ state, followed by an $\pi \pi^{*}\left(\mathrm{~S}_{2}\right)$ and an $n \pi^{*}\left(\mathrm{~S}_{3}\right)$ state (see Table 4). Thus, the nature of all three excited states change as one goes from thiophenol[ ${ }^{[23]}$ to the thione isomer $\mathbf{2 4}$. For $\mathbf{2 4}$ and $\mathbf{2 5}$ the most intense band is due to a transition to a $\pi \pi^{*}$ state, for all wavefunctions (see Tables 3 and 4).

\section{Conclusions}

The first four singlet states of thiones $\mathbf{2 4}$ and $\mathbf{2 5}$ have been studied using several types of uncontracted MR-CI wavefunctions, with the mixed aug-cc-PVDZ $(\mathrm{C}, \mathrm{H}) /$ aug-cc-pVTZ(S) basis set. Excitation energies $(\Delta \mathrm{E})$, oscillator strengths $(f)$ and nature of the excited states have been investigated. The basis set effect has also been taken into account. It is the first time that the excited states of $\mathbf{2 4}$ and $\mathbf{2 5}$ are studied.

The largest MR-CI calculation has been here performed for $\mathbf{2 5}$, due to its $\mathrm{C}_{2 \mathrm{v}}$ symmetry, achieving $\sim 3.6 \times 10^{8} \mathrm{CSFs}$. The change from thiophenol to 25 largely decreases the excitation energy of $\mathrm{S}_{1}$ (based on previous CASPT2/aug-cc-pVTZ ${ }^{[23]}$ and the $\Delta \mathrm{E}(+\mathrm{Q})$ results, see Table 3 ). In the case of $S_{2}$, the effect is opposite but much smaller, increasing its excitation energy by at most $0.56 \mathrm{eV}$ (at the-w4(+Q) level, see Table 3 ). In the case of $\mathrm{S}_{3}$ the effect is almost negligible for the MR-CISD wavefunctions ( $w 2$ and $w 4$, see Table 3).

Although $\mathrm{S}_{1}$ of $25\left(1^{1} \mathrm{~A}_{2}\right)$ is in the visible region (with $\Delta \mathrm{E}(+\mathrm{Q})$ from $\sim 2.0$ to $2.4 \mathrm{eV}$, see Table 3), the $1^{1} \mathrm{~A}_{1} \rightarrow 1^{1} \mathrm{~A}_{2}$ transition is dipole forbidden, by symmetry. The experimental threshold required for photochemical transformations of $\mathbf{2 5}, \lambda<332 \mathrm{~nm}\left(3.73 \mathrm{eV}^{[2]}\right)$ is, according to our results, consistent with a transition within the first band. Therefore, some 
intensity gain is expected to be taking place around $3.73 \mathrm{eV}$, due to vibronic and/or spin-orbit coupling mechanisms. Intensity borrowing from the nearby band cannot be ruled out.

Only the nature of $\mathrm{S}_{3}$ (a $\pi \pi^{*}$ state) is maintained as thiophenol changes to $\mathbf{2 5}$, with both $\mathrm{S}_{1}$ (also a $\pi \pi^{*}$ state) and $\mathrm{S}_{2}\left(\right.$ an $\mathrm{n}_{\pi} \sigma^{*}$ ) excited states changing their nature to $n \pi^{*}$. On the other hand, for 24 the nature of the three first excited singlet states is $n \pi^{*}, \pi \pi^{*}$ and $n \pi^{*}$, respectively. Thus, the nature of $S_{1}$ to $S_{3}$ changes upon the thiophenol $\rightarrow \mathbf{2 4}$ isomerization.

The $\Delta \mathrm{E}$ values decrease largely with the thiophenol $\rightarrow \mathbf{2 4}$ isomerization (again based on previous CASPT2/aug-cc-pVTZ ${ }^{[23]}$ and $\Delta \mathrm{E}(+\mathrm{Q})$ results), especially for $\mathrm{S}_{1}$. The only exception is observed for $\mathrm{S}_{3}$ with $w 3$ (see Table 4 ). The $\Delta \mathrm{E}(+\mathrm{Q})$ values, along with the computed $f$ values (although very small), are in line with the observation from ref. ${ }^{[2]}$ that $\mathbf{2 4}$ is the sole isomer absorbing in the visible region. The experimental threshold for the photochemical transformations of $24(\lambda<427 \mathrm{~nm}=2.90 \mathrm{eV})$ is consistent with excitation within the first band, associated with the $1^{1} \mathrm{~A}^{\prime \prime}\left(\right.$ an $\left.\mathrm{n} \pi^{*}\right)$ state.

Despite the relatively large differences obtained for the $f$ values computed with different wavefunctions, the $S_{0} \rightarrow S_{3}$ transition of $\mathbf{2 5}$ remains the most intense for all wavefunctions studied for this molecule. The same holds for the $S_{0} \rightarrow S_{2}$ transition of 24 .

Larger uncontracted MR-CI calculations still need to be performed (e.g., MR-CISD based on a set of CAS(12,11) reference CSFs), when they become computationally feasible, to see how the aforementioned results are affected. Alternatively, the effect of using more accurate extensivity corrections, as multi-reference average quadratic, MR-AQCC, might be evaluated in relation to alterations in the excited states' properties. ${ }^{[29]}$

\section{Acknowledgements}

I. R. acknowledges the Portuguese Foundation for Science and Technology (FCT) for an Investigador FCT grant. R.F. and I. R. thank FCT for financial support to the Coimbra Chemistry Centre (CQC, Research Unit UI0313/QUI/2020) co-funded by FEDER/COMPETE 2020-EU. E.V. thanks the Brazilian agency CNPq (Grant Number 303884/2018-5 and 423112/2018-0) for support. The remaining authors are grateful to the Brazilian agencies CAPES and FINEP for financial support. They are also thankful to the CESUP/UFRGS supercomputing center for the computational facilities. 


\section{References}

[1] A. H. Al-Muhtaseb, M. Altarawneh, M. H. Almatarneh, J Comput. Chem. 2011, 32, 2708. https://doi.org/10.1002/jcc.21852.

[2] I. Reva, M. J. Nowak, L. Lapinski, R. Fausto, Phys. Chem. Chem. Phys. 2015, 17, 4888. https://doi.org/10.1039/C4CP04125A.

[3] G. P. Rodrigues, T. M. L. de Lima, R. B. de Andrade, E. Ventura, S. A. do Monte, M. Barbatti, J. Phys. Chem. A 2019, 123, 1953. https://doi.org/10.1021/acs.jpca.8b12482.

[4] V. C. de Medeiros, R. B de Andrade, G. P. Rodrigues, G. F. Bauerfeldt, E. Ventura, M. Barbatti, S. A. do Monte, J. Chem. Theory Comput. 2018, 14, 4844. https://doi.org/10.1021/acs.jctc.8b00457.

[5] V. C. de Medeiros, R. B de Andrade, E. F. V. Leitão, E. Ventura, G. F. Bauerfeldt, M. Barbatti, S. A. do Monte, J. Am. Chem. Soc. 2016, 138, 272. https://doi.org/10.1021/jacs.5b10573.

[6] V. C. de Medeiros, S. A. do Monte, E. Ventura, RSC Adv. 2014, 4, 64085. https://doi.org/10.1039/C4RA10567B.

[7] G. P. Rodrigues, E. Ventura, S. A. do Monte, M. Barbatti, J. Phys. Chem. A 2014, 118, 12041. https://doi.org/10.1021/jp507681g.

[8] G. P. Rodrigues, J. R. Lucena Jr., E. Ventura, S. A. do Monte, I. Reva, R. Fausto, J. Chem. Phys. 2013, 139, 204302. https://doi.org/10.1063/1.4832376.

[9] V. C. de Medeiros, E. Ventura, S. A. do Monte, Chem. Phys. Lett. 2012, 546, 30. https://doi.org/10.1016/j.cplett.2012.07.037.

[10] J. R. Lucena Jr., E. Ventura, S. A. do Monte, R. C. M. U. Araújo, M. N. Ramos, J. Chem. Phys. 2007, 127, 164320. https://doi.org/10.1063/1.2800020.

[11] S. R. Langhoff, E. R. Davidson, Int. J. Quantum Chem. 1974, 8, 61. https://doi.org/10.1002/qua.560080106.

[12] P. J. Bruna, S. D. Peyerimhoff, R. J. Buenker, Chem. Phys. Lett. 1980, 72, 278. https://doi.org/10.1016/0009-2614(80)80291-0.

[13] P. G. Szalay, T. Müller, G. Gidofalvi, H. Lischka, R. Shepard, Chem. Rev. 2012, 112, 108. https://doi.org/10.1021/cr200137a.

[14] A. Bunge, J. Chem. Phys. 1970, 53, 20. https://doi.org/10.1063/1.1673766.

[15] R. Shepard, I. Shavitt, R, M. Pitzer, D. C. Comeau, M. Pepper, H. Lischka, P. G. Szalay, R. Ahlrichs, F. B Brown, J. Zhao, Int. J. Quantum Chem. 1988, 34, 149. https://doi.org/10.1002/qua.560340819.

[16] H. Lischka, R. Shepard, I. Shavitt, R. M. Pitzer, M. Dallos, T. Müller, P. G. Szalay, F. B. Brown, R. Ahlrichs, H. J. Böhm, et. al. COLUMBUS, an Ab Initio Electronic Structure Program, Release 7.0. www.univie.ac.at/columbus (accessed March 10, 2020).

[17] H. Lischka, R. Shepard, R. M. Pitzer, I. Shavitt, M. Dallos, T. Müller, P. G. Szalay, M. Seth, G. S. Kedziora, S. Yabushita, Z. Zhang. Phys. Chem. Chem. Phys. 2001, 3, 664. https://doi.org/10.1039/B008063M.

[18] H. Lischka, R. Shepard, F. B. Brown, I. Shavitt, Int. J. Quantum Chem. 1981, 20, 91. https://doi.org/10.1002/qua.560200810.

[19] T. Helgaker, H. J. Aa. Jensen, P. Jørgensen, J. Olsen, K. Ruud, H. Ågren, T. Andersen, K. L. Bak, V. Bakken, O. Christiansen, et. al. DALTON, an Ab Initio Electronic Structure Program, Release 1.0. 1997. 
[20] T. H. Dunning, J. Chem. Phys. 1989, 90, 1007. https://doi.org/10.1063/1.456153.

[21] R. A. Kendall, T. H. Dunning, R. J. Harrison, J. Chem. Phys. 1992, 96, 6796. https://doi.org/10.1063/1.46256.

[22] D. E. Woon, T. H. Dunning. J. Chem. Phys. 1993, 98, 1358. https://doi.org/10.1063/1.464303.

[23] M. N. R. Ashfold, G. A. King, D. Murdock, M. G. D. Nix, T. A. A. Oliver, A. G. Sage, Phys. Chem. Chem. Phys. 2010, 12, 1218. https://doi.org/10.1039/B921706A.

[24] L. Zhang, D. G. Truhlar, S. Sun, Phys. Chem. Chem. Phys. 2018, 20, 28144. https://doi.org/10.1039/C8CP05215H.

[25] H. S. You, S. Han, J. S. Lim, S. K. Kim, J. Phys. Chem. Lett. 2015, 6, 3202. https://doi.org/10.1021/acs.jpclett.5b01420.

[26] T. S. Venkatesan, S. G. Ramesh, W. Domcke, J. Chem. Phys. 2012, 136, 174312. https://doi.org/10.1063/1.4709608.

[27] Guang-Shuang-Mu Lin, C. Xie, D. Xie, J. Phys. Chem. A 2017, 121, 8432. https://doi.org/10.1021/acs.jpca.7b09070.

[28] H. Lischka, D. Nachtigallová, A. J. A. Aquino, P. G. Szalay, F. Plasser, F. B. C. Machado, M. Barbatti, Chem. Rev. 2018, 118, 7293. https://doi.org/10.1021/acs.chemrev.8b00244.

[29] S.A. do Monte, M. Dallos, T. Müller, H. Lischka, Collect. Czech. Chem. Commun. 2003, 68, 447. https://doi.org/10.1135/cccc20030447. 
New properties of two new thione isomers of thiophenol

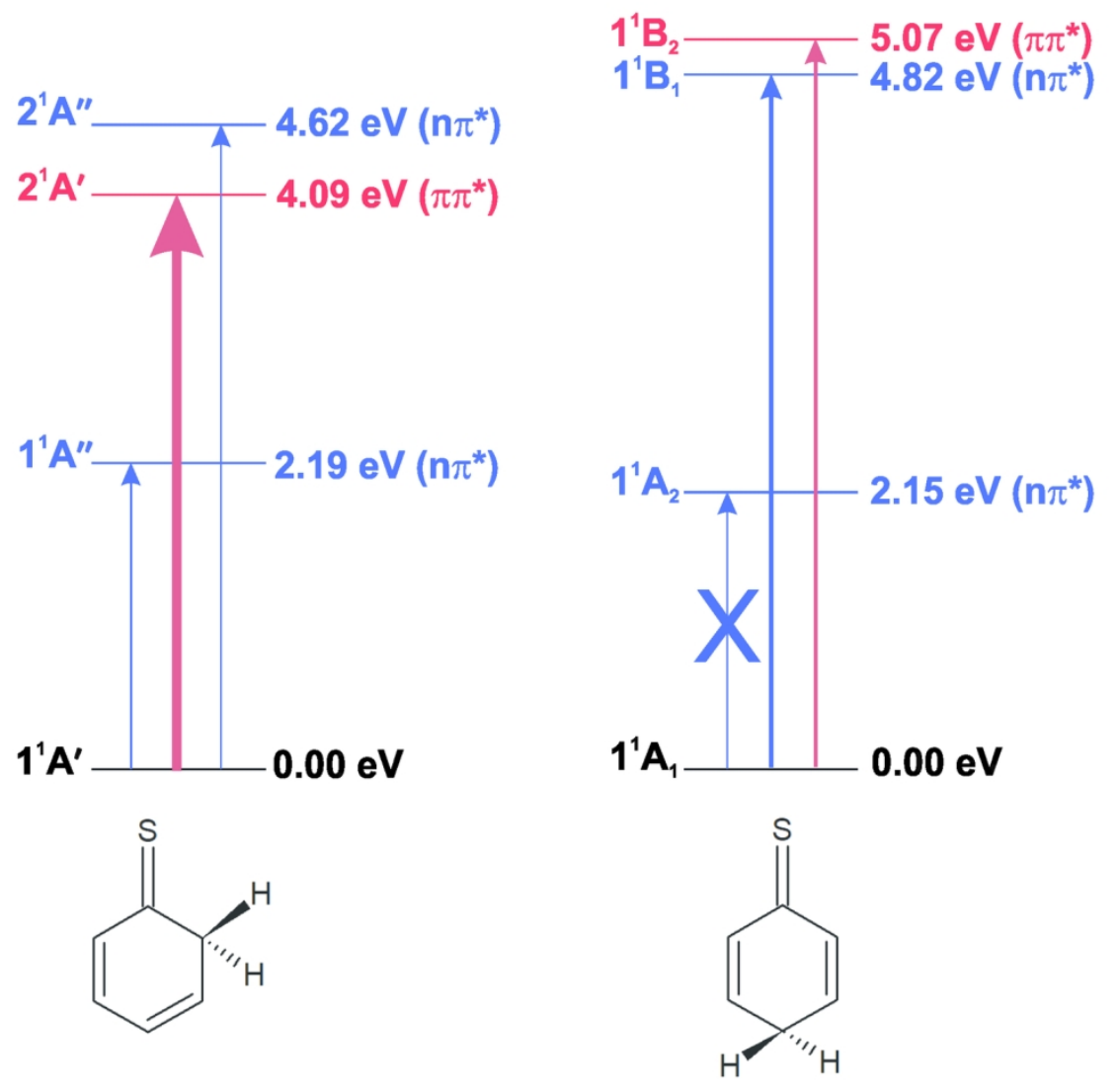

A correct description of excited states properties is the first step towards a deep understanding of the photochemistry of compounds. This article show accurate results for some excited states of two new thione isomers of thiophenol, which can help to discriminate between these three species as well to follow spectroscopically their interconversions. 


\section{Supporting Information}

\section{A comparative multi-reference configuration interaction study of the low-lying states of two thione isomers of thiophenol}

Filipe Belarmino de Lima, ${ }^{1}$ Gessenildo Pereira Rodrigues, ${ }^{2}$ Juracy Regis de Lucena Junior, ${ }^{3}$ Elizete Ventura, ${ }^{1}$ Rui Fausto, ${ }^{4}$ Igor Reva, ${ }^{4 *}$ Silmar Andrade do Monte ${ }^{1 *}$

${ }^{1}$ Departamento de Química, CCEN, Universidade Federal da Paraíba, 58059-900, João Pessoa, Brazil ${ }^{2}$ Faculdade Rebouças, 58406-040, Campina Grande, Brazil

${ }^{3}$ Departamento de Química, Universidade Estadual da Paraíba, 58429-500, Campina Grande, Brazil ${ }^{4}$ CQC-Departamento de Química, Universidade de Coimbra, 3004-535, Coimbra, Portugal

* Corresponding authors.

e-mails: reva@qui.uc.pt, silmar@quimica.ufpb.br

Contents: $\quad$ Page

Table S1. Active orbitals of Cyclohexa-2,5-diene-1-thione (thione 25) 2

Table S2. Active orbitals of Cyclohexa-2,4-diene-1-thione (thione 24) 3

Occupation numbers of the active orbitals of thiones 25 and 24, obtained from 4 state-average CASSCF calculations

Occupation numbers of the active orbitals of thiones 25 and 24 in the four states, obtained at the $w 1$ level 
Table S1. Active orbitals of Cyclohexa-2,5-diene-1-thione (thione 25).

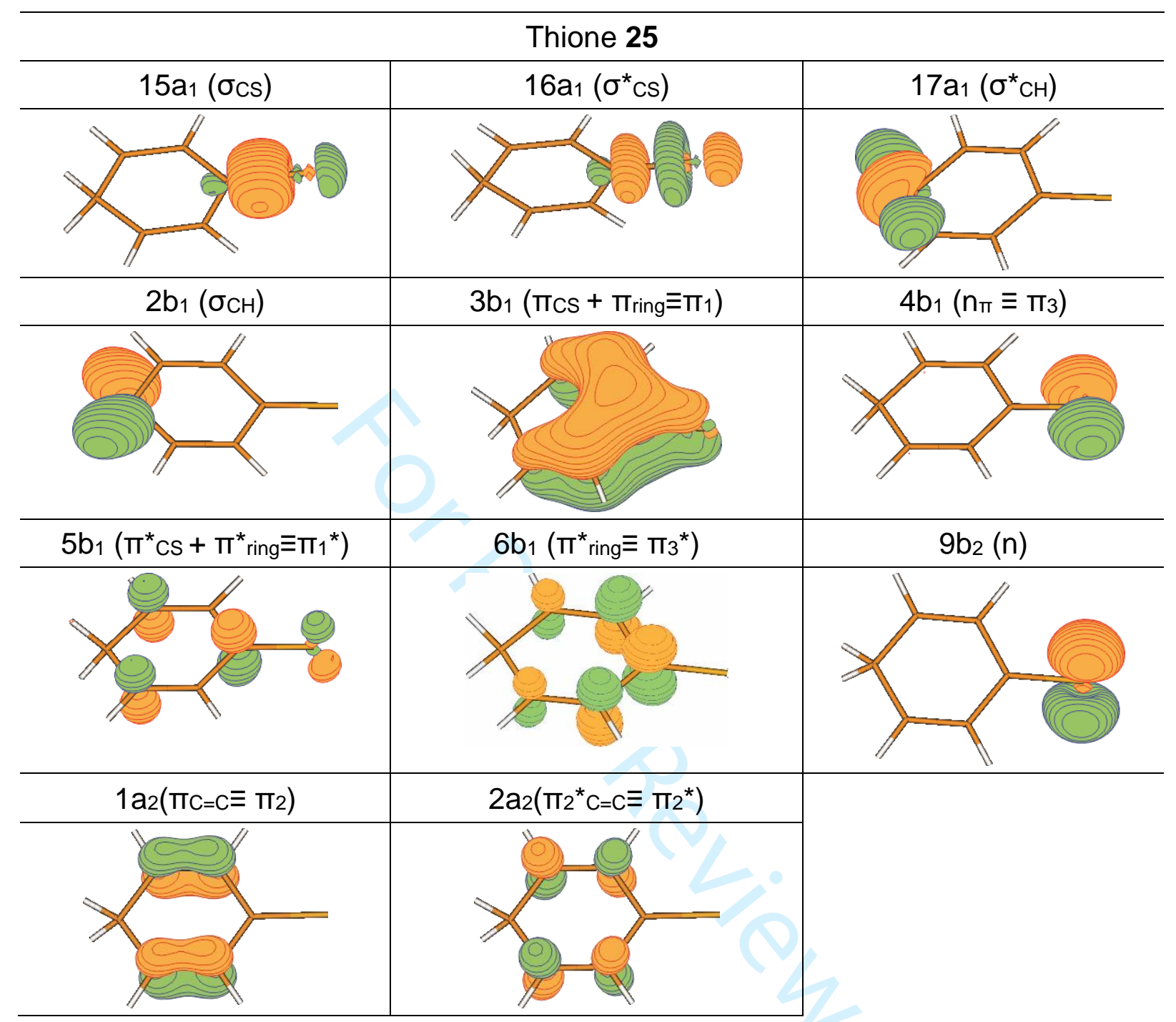

Contour values: For the $3 b_{1}$ orbital a contour value of 0.05 has been used, while for the remaining orbitals a value of 0.10 has been used.

At the SCF level, the electronic configuration is the following:

$\left(1 a_{1}\right)^{2}\left(2 a_{1}\right)^{2}\left(3 a_{1}\right)^{2}\left(1 b_{2}\right)^{2}\left(4 a_{1}\right)^{2}\left(2 b_{2}\right)^{2}\left(5 a_{1}\right)^{2}\left(6 a_{1}\right)^{2}\left(7 a_{1}\right)^{2}\left(1 b_{1}\right)^{2}\left(3 b_{2}\right)^{2}\left(8 a_{1}\right)^{2}\left(9 a_{1}\right)^{2}\left(4 b_{2}\right)^{2}\left(10 a_{1}\right)^{2}$ $\left(5 b_{2}\right)^{2}\left(11 a_{1}\right)^{2}\left(12 a_{1}\right)^{2}\left(6 b_{2}\right)^{2}\left(13 a_{1}\right)^{2}\left(2 b_{1}\right)^{2}\left(7 b_{2}\right)^{2}\left(14 a_{1}\right)^{2}\left(8 b_{2}\right)^{2}\left(15 a_{1}\right)^{2}\left(3 b_{1}\right)^{2}\left(a_{2}\right)^{2}\left(9 b_{2}\right)^{2}\left(4 b_{1}\right)^{2}$ $\left(16 a_{1}\right)^{0}\left(5 b_{1}\right)^{0}\left(10 b_{2}\right)^{0}\left(6 b_{1}\right)^{0}\left(17 a_{1}\right)^{0}\left(11 b_{2}\right)^{0}\left(18 a_{1}\right)^{0}\left(19 a_{1}\right)^{0}\left(2 a_{2}\right)^{0}\left(7 b_{1}\right)^{0}\left(20 a_{1}\right)^{0}\left(12 b_{2}\right)^{0}\left(21 a_{1}\right)^{0}\left(3 a_{2}\right)^{0}$

The first eleven (underlined) are core orbitals. The orbitals in bold are those included in the CAS space; the active orbitals shown in black and red correspond to occupied and virtual, respectively. 
Table S2. Active orbitals of Cyclohexa-2,4-diene-1-thione (thione 24).

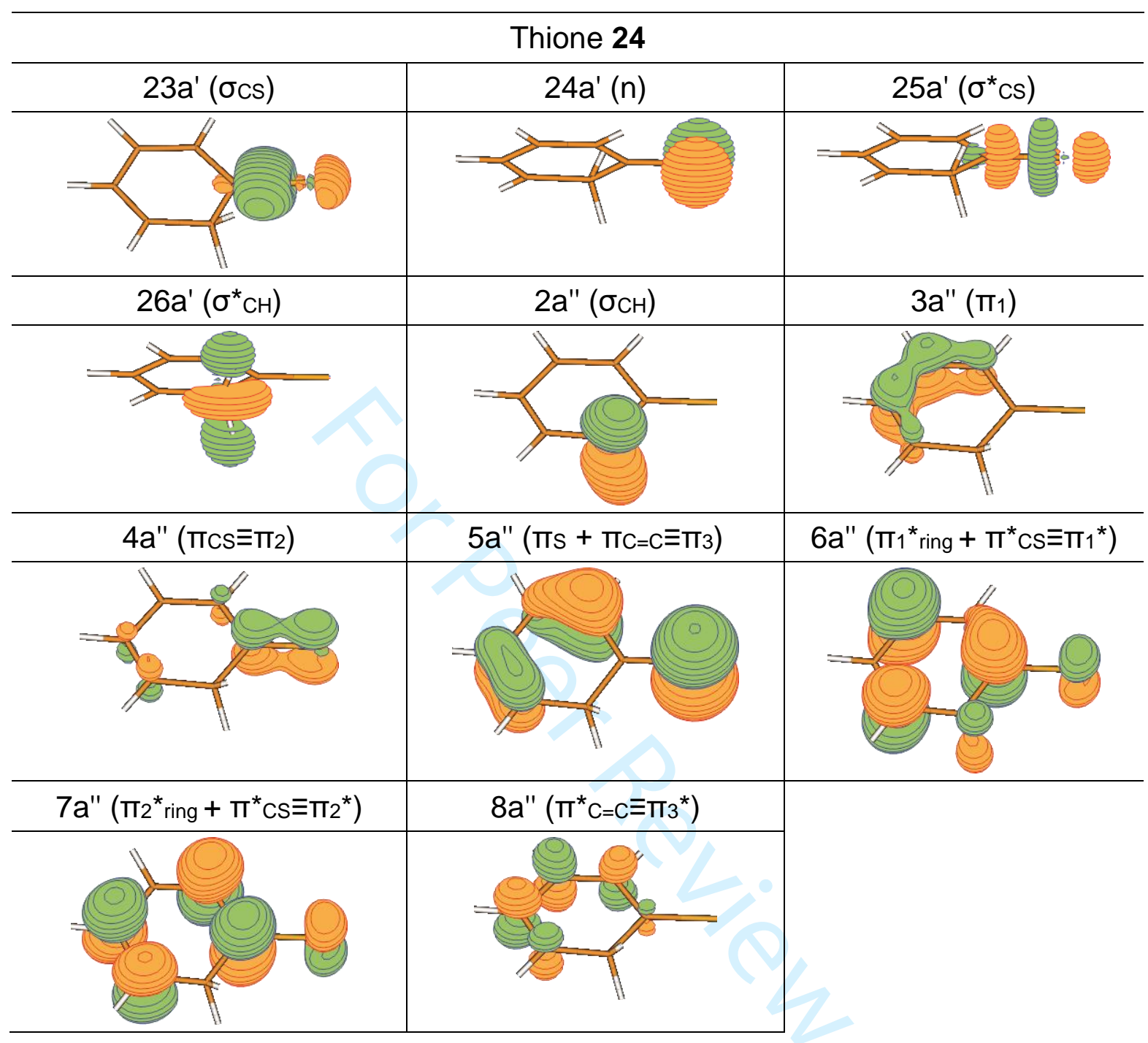

Contour values: For the $5 \mathrm{a}^{\prime \prime}, 6 \mathrm{a}$ " and $7 \mathrm{a}$ " orbitals a contour value of 0.05 has been used, while for the remaining orbitals a value of 0.10 has been used.

At the SCF level, the electronic configuration is the following:

$\left(1 a^{\prime}\right)^{2}\left(2 a^{\prime}\right)^{2}\left(3 a^{\prime}\right)^{2}\left(4 a^{\prime}\right)^{2}\left(5 a^{\prime}\right)^{2}\left(6 a^{\prime}\right)^{2}\left(7 a^{\prime}\right)^{2}\left(8 a^{\prime}\right)^{2}\left(9 a^{\prime}\right)^{2}\left(1 a^{\prime \prime}\right)^{2}\left(10 a^{\prime}\right)^{2}\left(11 a^{\prime}\right)^{2}\left(12 a^{\prime}\right)^{2}\left(13 a^{\prime}\right)^{2}\left(14 a^{\prime}\right)^{2}$ $\left(15 a^{\prime}\right)^{2}\left(16 a^{\prime}\right)^{2}\left(17 a^{\prime}\right)^{2}\left(18 a^{\prime}\right)^{2}\left(19 a^{\prime}\right)^{2}\left(2 a^{\prime \prime}\right)^{2}\left(20 a^{\prime}\right)^{2}\left(21 a^{\prime}\right)^{2}\left(22 a^{\prime}\right)^{2}\left(23 a^{\prime}\right)^{2}\left(3 a^{\prime \prime}\right)^{2}\left(4 a^{\prime \prime}\right)^{2}\left(24 a^{\prime}\right)^{2}\left(5 a^{\prime \prime}\right)^{2}$ $\left(6 a^{\prime \prime}\right)^{0}\left(25 a^{\prime}\right)^{0}\left(26 a^{\prime}\right)^{0}\left(27 a^{\prime}\right)^{0}\left(7 a^{\prime \prime}\right)^{0}\left(28 a^{\prime}\right)^{0}\left(29 a^{\prime}\right)^{0}\left(30 a^{\prime}\right)^{0}\left(8 a^{\prime \prime}\right)^{0}$

The first eleven (underlined) are core orbitals. The orbitals in bold are those included in the CAS space; the active orbitals shown in black and red correspond to occupied and virtual, respectively. 
Occupation numbers of the active orbitals obtained from state-average CASSCF calculations (in descending order):

Cyclohexa-2,5-diene-1-thione (thione25):

\begin{tabular}{|c|c|c|c|c|c|c|c|c|c|c|}
\hline $2 \mathrm{~b}_{1}$ & $15 \mathrm{a}_{1}$ & $3 \mathrm{~b}_{1}$ & $1 \mathrm{a}_{2}$ & $4 \mathrm{~b}_{1}$ & $9 \mathrm{~b}_{2}$ & $5 \mathrm{~b}_{1}$ & $2 \mathrm{a}_{2}$ & $6 \mathrm{~b}_{1}$ & $16 \mathrm{a}_{1}$ & $17 \mathrm{a}_{1}$ \\
\hline 1.9936 & 1.9775 & 1.9098 & 1.6943 & 1.6978 & 1.4995 & 0.7706 & 0.3405 & 0.0879 & 0.0229 & 0.0055 \\
\hline
\end{tabular}

The last five are anti-bonding orbitals, with occupation numbers smaller than 1.0. The numbering of the $\pi$ orbitals shown in Table $S 1$ is given according to their occupation numbers. The subscripts in the plots (for instance, CS + ring) refer to the localization of the orbitals.

Cyclohexa-2,4-diene-1-thione (thione 24):

\begin{tabular}{|c|c|c|c|c|c|c|c|c|c|c|}
\hline $2 a^{\prime}$ & $23 a^{\prime}$ & $3 a^{\prime \prime}$ & $4 a^{\prime \prime}$ & $5 a^{\prime \prime}$ & $24 a^{\prime}$ & $6 a^{\prime \prime}$ & $7 a^{\prime \prime}$ & $8 a^{\prime \prime}$ & $25 a^{\prime}$ & $26 a^{\prime}$ \\
\hline 1.9928 & 1.9759 & 1.9138 & 1.8044 & 1.6046 & 1.4991 & 0.7997 & 0.3019 & 0.0777 & 0.0245 & 0.0056 \\
\hline
\end{tabular}

The last five are anti-bonding orbitals, with occupation numbers smaller than 1.0. The numbering of the $\pi$ orbitals shown in Table $\mathrm{S} 2$ is given according to their occupation numbers. The subscripts in the plots (for instance, $\mathrm{S}+\mathrm{C}=\mathrm{C}$ ) refer to the localization of the orbitals. 
Occupation numbers of the active orbitals of thiones $\mathbf{2 5}$ and $\mathbf{2 4}$ in the four states, obtained at the $w 1$ level.

Cyclohexa-2,5-diene-1-thione (thione 25):

\begin{tabular}{|c|c|c|c|c|c|c|c|c|c|c|c|}
\hline State & $2 \mathrm{~b}_{1}$ & $15 \mathrm{a}_{1}$ & $3 \mathrm{~b}_{1}$ & $1 \mathrm{a}_{2}$ & $4 \mathrm{~b}_{1}$ & $9 \mathrm{~b}_{2}$ & $5 \mathrm{~b}_{1}$ & $2 \mathrm{a}_{2}$ & $6 \mathrm{~b}_{1}$ & $16 \mathrm{a}_{1}$ & $17 \mathrm{a}_{1}$ \\
\hline $1^{1} \mathrm{~A}_{1}$ & 1.9834 & 1.9631 & 1.9401 & 1.9186 & 1.8782 & 1.9808 & 0.1391 & 0.0759 & 0.0526 & 0.0386 & 0.0116 \\
\hline $1^{1} \mathrm{~A}_{2}$ & 1.9839 & 1.9654 & 1.9764 & 1.9138 & 1.9372 & 1.0043 & 1.0031 & 0.0887 & 0.0644 & 0.0351 & 0.0117 \\
\hline $1^{1} \mathrm{~B}_{1}$ & 1.9825 & 1.9644 & 1.9654 & 1.7520 & 1.9103 & 1.0043 & 0.4527 & 0.8236 & 0.0787 & 0.0352 & 0.0113 \\
\hline $1^{1} \mathrm{~B}_{2}$ & 1.9828 & 1.9661 & 1.9265 & 1.2977 & 1.4646 & 1.9814 & 0.8783 & 0.3690 & 0.0605 & 0.0350 & 0.0120 \\
\hline
\end{tabular}

Cyclohexa-2,4-diene-1-thione (thione 24):

\begin{tabular}{|c|c|c|c|c|c|c|c|c|c|c|c|}
\hline State & $2 a^{\prime \prime}$ & $23 a^{\prime}$ & $3 a^{\prime \prime}$ & $4 a^{\prime \prime}$ & $5 a^{\prime \prime}$ & $24 a^{\prime}$ & $6 a^{\prime \prime}$ & $7 a^{\prime \prime}$ & $8 a^{\prime \prime}$ & $25 a^{\prime}$ & $26 a^{\prime}$ \\
\hline $1^{1} \mathrm{~A}^{\prime}$ & 1.9831 & 1.9802 & 1.9404 & 1.9196 & 1.8697 & 1.9625 & 0.1413 & 0.0816 & 0.0514 & 0.0392 & 0.0138 \\
\hline $1^{1} \mathrm{~A}^{\prime \prime}$ & 1.9839 & 1.9649 & 1.9759 & 1.9413 & 1.9048 & 1.0043 & 1.0023 & 0.0993 & 0.0593 & 0.0354 & 0.0129 \\
\hline $2^{1} \mathrm{~A}^{\prime}$ & 1.9780 & 1.9713 & 1.9364 & 1.9073 & 1.5330 & 1.9610 & 0.4826 & 0.0995 & 0.0471 & 0.0403 & 0.0171 \\
\hline $2^{1} \mathrm{~A}^{\prime \prime}$ & 1.9833 & 1.9645 & 1.9701 & 1.9244 & 1.6128 & 1.0043 & 0.7416 & 0.6589 & 0.0723 & 0.0359 & 0.01264 \\
\hline
\end{tabular}

